study would be "paranoid schizophrenia with a presentation modified by cannabis use".

South Wales Forensic Psychiatric Service

Huw Thomas

Casewell Clinic

Glanrhyd Hospital

Bridgend

Mid-Glamorgan CF31 4LN

Wales

\section{Conversion mnemonic}

SIR: I enjoyed reading the articles by Mace (Journal, September 1992, 161, 369-389). However, the mnemonic I have devised as a teaching tool about 'conversion' contains possibly important elements which were not addressed in the articles:

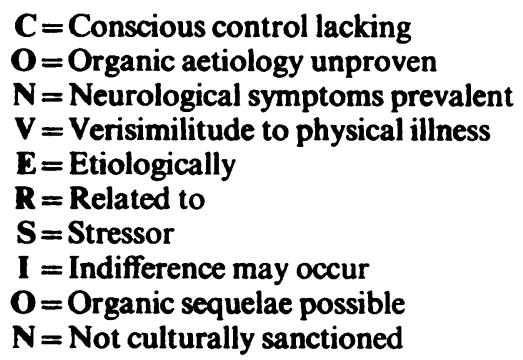

The elements 'indifference', 'organic sequelae', and 'not culturally sanctioned' were largely omitted in the discussion by Mace.

La belle indifference (inappropriate lack of emotion or concern for the implications of one's disability (Stedman, 1990)) was, perhaps, the most striking omission in a historical review of conversion hysteria. This concept, like the concept of conversion itself, appears to have withstood the test of time; although the implications of the attitude described by la belle indifference remain to be clarified. Perhaps la belle indifference refers to the physician's attitude when an organic aetiology cannot be proven, similar to the suggestion by Mace that 'conversion' refers to a change in the physician's attitude towards a patient who defies traditional diagnosis.

Possible organic sequelae are important in considering the natural history of untreated conversion symptoms. Phenomena such as contractures, disuse atrophy, decubiti, and invalidism all demonstrate that patients with conversion can, ultimately, progress to serious physical illness.

Classical descriptions of conversion symptoms usually exclude culturally sanctioned behaviour. Otherwise, behaviour such as 'glossolalia' ('speaking in tongues' associated with certain religious sects
(Kaplan \& Sadock, 1989)) might further confuse the issue.

The concept of conversion will certainly continue to undergo a fascinating evolution. Specific disorders without proven organic aetiology (e.g. "chronic fatigue syndrome' (Goldman, 1992)) are also testing current diagnostic boundaries of neurology and psychiatry.

Goldman, M. B. (1992) Neuropsychiatric features of endocrine disorders. In The American Psychiatric Press Texibook of Neuropsychiatry (2nd edition) (eds S. C. Yudosfski \& R. E. Hales), p. 528. Washington: American Psychiatric Press.

KAFLAN, H. I. \& SADOCK, B. J. (1989) Diagnosis and psychiatry: examination of the psychiatric patient. In Comprehensive Textbook of Psychiatry/V (5th edition) (eds H. I. Kaplan \& B. J. Sadock), p. 472. Baltimore: Williams and Wilkins.

Stedman (1990) Stedman's Medical Dictionary, 25th edition. Baltimore: Williams and Wilkins.

Eve J. WISEMAN

Department of Psychiatry

University of Arkansas for Medical Sciences

Special Treatment Section

Little Rock Veterans' Administration Hospital

Arkansas 72205, USA

\section{Gender difierence of schizophrenia in seasonal stmiscions in Scotland}

SIR: Using a national sample from England and Wales, we reported that an excess of first admissions was present in summer months in female but not male schizophrenics (Takei et al, Journal, October $1992,161,506-511)$. Since this summer excess of admissions was also demonstrated in patients with a diagnosis of mania, we suggested that female schizophrenia has some aetiological or precipitating factor in common with mania.

To determine whether the gender difference in season of admissions in schizophrenia is reproducible, we obtained data on all first admissions for schizophrenia in Scotland between 1961 and 1990. The diagnosis of schizophrenia was coded using the 7th revision of the International Classification of Diseases (ICD) (World Health Organization) for those admitted between 1961 and 1967, ICD-8 for those admitted between 1968 and 1979, and ICD-9 for those admitted between 1980 and 1990 . We tested the cyclical variation in admissions using the Edwards' method, the advantages of which we have previously discussed (Takei et al, 1992).

The results revealed that there was a significant cyclical seasonality in schizophrenic admissions $\left(n=14964, \chi^{2}=12.26\right.$, d.f. $\left.=2, P<0.005\right)$. When the sexes were examined separately, a highly significant cyclical seasonality was found in female $(n=6875$, 
$\chi^{2}=11.08$, d.f. $\left.=2, P<0.005\right)$ but not male schizophrenics $\left(n=8089, \chi^{2}=2.93\right.$, d.f. $=2$, NS $)$. The estimated peak for female schizophrenics, and for both sexes taken together, was early May.

The existence of significant seasonality in part supports the findings of our England and Wales study. This study is, however, weak, in that we were not, at that time, able to obtain the data on first admissions for mania in Scotland, and could not compare the seasonal variation of female schizophrenics with that of manics. Nevertheless, the gender difference in season of admissions in schizophrenia persisted in a second large data set. However, the estimated peak of admissions in this study precedes by two months that of our English data, which showed a July peak of admissions for female schizophrenics. Although the reason for this discrepancy is unclear, some geographical or climatic factor(s) may play a role. The delay in the peak of admissions in England and Wales compared with Scotland is at odds with the idea that increasing warmth or lengthened daylight is responsible for the increase in admissions.

We thank Dr S. K. Cole, Scottish Health Service, Common Services Agency, Edinburgh, for providing us with data.

EDWARDS, J. H. (1961) The recognition and estimation of cyclic trends. Annals of Human Genetics, 25, 83-86.

TAKel, N., O'Callaghan, E., Sham, P., et al (1992) Seasonality of admissions in the psychoses: effect of diagnosis, sex, and age at onset. British Journal of Psychiatry. 161, 506-511.

R. M. MURRAY
Department of Psychological Medicine
Institute of Psychiatry \& King's College Hospital
De Crespigny Park
London SES 8AF

\section{Estimating premorbid IQ in schizophrenia}

SIR: Crawford et al (Journal, July 1992, 161, 69-74) reported comparisons between controls and schizophrenic samples on the Wechsler Adult Intelligence Scale (WAIS; Wechsler, 1958) scores and National Adult Reading Test (NART; Nelson, 1982) scores; community resident and long-stay hospital patients had significantly lower verbal and performance WAIS scores than controls, whereas only long-stay patients had poorer NART scores than controls. They discussed the efficacy of using the NART to estimate premorbid IQ but no conclusions were reached as to whether the low scores in the longstay sample were a true indication of low premorbid IQ or whether "NART performance was impaired by onset of disease". We wish to draw attention to a methodological point regarding the study design and to a general problem in using a reading test such as the NART in disorders beginning in early adult life.

In order to draw conclusions on the effects of schizophrenia on test results, cases and controls should have been comparable, other than for the presence of schizophrenia. The absence of long-term in-patients in the control group for long-stay patients may have introduced bias; poor intellectual functioning may make life in long-stay care more likely, regardless of schizophrenia. Also, the effects of neuroleptic drugs, present only in the cases, may have attenuated test performance. If, as Crawford et al suggest, NART score and, to a lesser extent, WAIS verbal skills are relatively resistant to the ravages of neuropathology, compared with performance skills, they may also be resistant to the deleterious effects of neuroleptics. Drug dosages and test scores could be examined for such dose-response relationships.

Use of the NART as a measure of premorbid IQ has become increasingly common in studies of cognitive ageing and dementia, and has been extended to other conditions (Crawford, 1989). However, its applicability to schizophrenia and other disorders of early adulthood raises difficulties because the considered explanations of poor performance are not comprehensive. Knowledge of the acquisition of intellectual skills in the normal population forms a useful foundation for hypotheses concerning the disruptive effects of pathology. The view that intellectual functioning reaches a peak in the teenage years has been supplanted by the less rigid notion of significant advancement later, possibly much later, in life and of different age gradients for different skills. The empirical evidence is sparse concerning the acquisition of reading skills but suggests a substantial improvement for the general population through the late teenage years and early adulthood, perhaps continuing into middle life (Rodgers, 1986). One might predict similar gains for the NART. That statistically significant age gradients have not been reported (Crawford, 1989) may be due to relatively small samples and the inextricable confounding of age and cohort effects in cross-sectional study designs.

If an appreciable gain in reading skills occurs in the general population over the age range representing the maximum risk period for incidence of schizophrenia (or other disorders), patients may show a slower rate or cessation of improvement as illness impinges on their normal activities. Under this model, a relative deficit in NART performance reflects neither erosion of previously attained 